\title{
Photometric Calibrations after HIPPARCOS or: The Fine Structure of the HR-Diagram Revealed
}

\author{
Michel Grenon \\ Geneva Observatory, 1290 Sauverny, Switzerland
}

\begin{abstract}
The Hipparcos parallaxes allow us to revisit photometric calibrations in terms of $M_{v}$ and to evaluate the effects of the overall metallicity across the HR-diagram. For evolved stars the spread in mass remains the major and irreducible source of errors in the photometric $M_{v}$. If the relative locations of open cluster main-sequences are fully explained by the $\Delta M_{v}$ versus $\Delta[\mathrm{M} / \mathrm{H}]$ relation, the residual scatter in the lower main sequence appears due to additional parameters such as rotation or gravitational settling of the heavy elements. These cannot readily be studied by a purely photometric approach.
\end{abstract}

\section{Introduction}

The high accuracy of the parallaxes has induced most investigators to use Hipparcos astrometric instead of photometric parallaxes, combined with photometric estimates of parameters such as $T_{\text {eff }}, \log g$ and when available $[\mathrm{M} / \mathrm{H}]$. The major improvements in the delineation of the HR-diagram affect stars brighter than $M_{v}=2$, namely the red and blue giants. The detailed structure of the GB and RHB is now available provided age and metallicity effects are disentangled through photometric $[\mathrm{M} / \mathrm{H}]$ and $T_{\text {eff }}$. The first direct absolute luminosities of field AGB stars are also available in the $V-I$ range 1.7 to 4 . For the first time the dependence of $M_{v}$ on $[\mathrm{M} / \mathrm{H}]$ or $[\alpha / \mathrm{H}]$ for M giants may be deduced from TiO band intensities. In the $M_{v}$ range 2 to 7 , values of $M_{v t r i g}$ are precise enough to allow investigations of the fine structure of the main sequence, namely to check the presence of gaps and the change of slope and to evaluate the dependence of $M_{v}$ on $[\mathrm{M} / \mathrm{H}]$ and on the $\mathrm{He}$ abundance etc.

From colour combinations sensitive preferentially to $T_{\text {eff }}, \log g$ or $[\mathrm{M} / \mathrm{H}]$ variations, photometrists provide calibrations in terms of $T_{\text {eff }}, M_{v}$ and possibly $[\mathrm{M} / \mathrm{H}]$ of so-called temperature, luminosity and metallicity parameters, often with the implicit hypothesis that a one-to-one relation exists between $M_{v}$ and $\log g$ at given $T_{\text {eff }}$ across the HR-diagram.

\section{The calibrations in terms of $M_{v}$ or luminosity class}

Attempts to transform gravity and temperature parameters in terms of $M_{v}$ and $T_{\text {eff }}$, with $M_{v}$ calibrated against Hipparcos $M_{v}$, are invalid in all degenerate areas of the HR-diagram, i.e. in areas where a spread in mass exists at given luminosity and temperature. The classical calibration schemes are strictly valid 
for main-sequence and subgiant stars but not for luminous B and A stars, nor for most red giants.

The most critical area is that of the red giant clump and RHB, where the possible mass ranges from 0.6 to $2.5 M_{\odot}$ at given $L$ and $T_{\text {eff }}$. When Hipparcos Survey stars are used as luminosity calibrators, the mean mass $M_{\text {mean }}$ of RHB stars is $\sim 1.1 M_{\odot}$. For stars with masses $M_{*}$ different from $M_{\text {mean }}$, the photometric or spectroscopic calibrations lead to systematic errors in $M_{v}$ equal to $3.5 \log \left(M_{*} / M_{\text {mean }}\right)$. Through the mass, these errors are age dependent. In the case of the Hyades generation, $M_{v_{\text {phot }}}$ of clump stars are overestimated by at least $1.5 \mathrm{mag}$. The same limitations apply to calibrations in terms of $M_{v}$ of spectroscopic parameters such as the MK luminosity class or the Ca II K emission since they are also functions of the stellar gravity and not of the luminosity.

Since Cannon's work in 1973, the absolute magnitude of the clump, $M_{v} \sim 1$, was widely used to derive open cluster distances. The precise dependence of $M_{v \mathrm{RHB}}$ on $[\mathrm{M} / \mathrm{H}]$ for the field metal-poor RHBs was established by Gratton (1998) using Hipparcos parallaxes, by Twarog, Anthony-Twarog \& Bricker (1999) for intermediate metallicity RHBs and by Girardi et al. (1998) for solar to metal-rich stars. The dependence of $M_{I}$ (in the Cousins $I$ band) on age and $[\mathrm{M} / \mathrm{H}]$ is very weak, so the RHBs are now among the most reliable distance estimators and are applicable everywhere in the Galaxy.

Because of the HR-diagram degeneracy, astrometric parallaxes of red giants are preferred to photometric parallaxes at least up to $\sigma\left(M_{v \text { trig }}\right) \sim 1.0$. On the other hand, the photometric estimates of $[\mathrm{M} / \mathrm{H}]$ and $\mathcal{T}_{\text {eff }}$ are fully reliable although some scatter may be present due to $[\mathrm{CN} / \mathrm{Fe}]$ anomalies, mass and initial rotation.

\section{The distance to open clusters}

Van Leeuwen \& Hansen Ruiz (1997) found the Pleiades underluminous by $\sim 0.4$ mag with respect to the Hyades excluding an effect of a large He overabundance. A puzzling situation arose from the similarity of $[\mathrm{Fe} / \mathrm{H}]$ for the Pleiades and the Hyades, +0.026 and +.095 dex respectively, the difference being too small to explain the large observed $\Delta M_{v}$. As a result of large rotational velocities and the diversity of methods of analysis, the published spectroscopic $[\mathrm{Fe} / \mathrm{H}]$ values for the Pleiades show a scatter as large as that observed in the whole old disc population. With revised $[\mathrm{Fe} / \mathrm{H}]$, Mermilliod (2000) could explain most of the differences in cluster main-sequence positions as due to the dependence on metalabundance, but the Pleiades anomaly is not wholly removed.

From Geneva photometry on single stars $[\mathrm{M} / \mathrm{H}]=-0.080 \pm 0.023$ for the Pleiades and $+0.144 \pm 0.013$ for the Hyades, without noticeable internal scatter among cluster members. The general relation between $\Delta M_{v}$ and $[\mathrm{M} / \mathrm{H}]$ is defined over a broader range of $[\mathrm{M} / \mathrm{H}]$ using unevolved field $\mathrm{K}$ dwarfs with $M_{v} \geq 5$ :

$$
\Delta M_{v}=-1.67 \cdot \Delta[\mathrm{M} / \mathrm{H}]
$$

When applied to nearby open clusters, this relation exactly matches the relative location of cluster sequences around $1 M_{\odot}$, see Fig. 1. A unique relation between $M_{v}$ and the metallicity exists at given $T_{\text {eff }}$, at least for young to intermediate 
age stars. This is a strong indication of a close correlation between the helium content and the overall metallicity in the local Galactic disc.

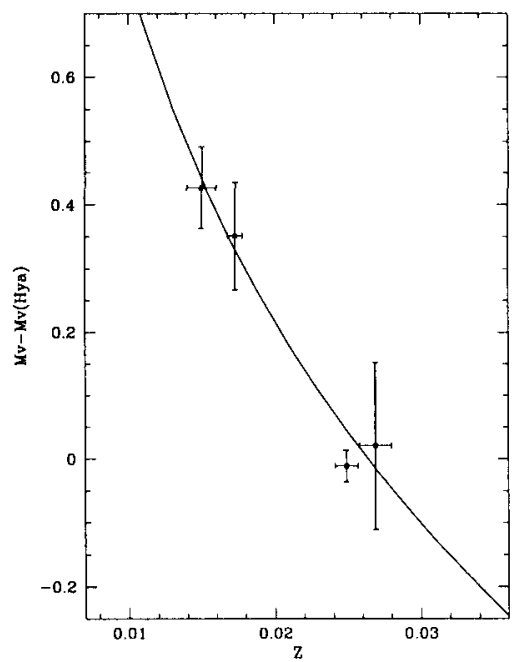

Figure 1. The departure from the Hyades main-sequence as a function of the heavy element fraction Z: for the Pleiades (top), NGC 2451 (middle), the Hyades and Praesepe (bottom). The solid line indicates the relation for field $\mathrm{K}$ dwarfs with $M_{v}$ from Hipparcos parallaxes and $\mathrm{Z}$ from Geneva photometry.

So the Pleiades He abundance is not exotic, with $\mathrm{Y} \sim 0.28$, and the luminosity of the unevolved main-sequence is exactly as expected from its $[\mathrm{M} / \mathrm{H}]$.

\section{The residual scatter in the main sequence luminosity}

When stars of all ages are considered, the lower main sequence remains rather thick, i.e. $\pm 0.3 \mathrm{mag}$, at given temperature and metallicity, cf. Fig. 2. Its width exceeds significantly the effects of errors in $M_{v \text { trig }}$, limited to 0.12 mag., and in $[\mathrm{M} / \mathrm{H}]$, i.e. 0.03 to 0.05 dex. A comparison between the shape of the Hyades sequence with that of field stars with large proper-motions which have ages from 4 to $10 \mathrm{Gyr}$, reveals a conspicuous difference of slope in the $M_{v}$ range 5 to 7 , but there is good agreement in the $M_{v}$ range 7 to 10, i.e. below the Mazzitelli gap around $M_{v}=7$. If the residual spread in $M_{v}$ is purely an age effect, this limits the achievable accuracy on photometric $M_{v}$ to at best $\pm 0.25 \mathrm{mag}$.

Whereas the stellar activity, the surface rotation or the microturbulence may be deduced from high dispersion analysis, the effect of internal rotation and of heavy element diffusion are beyond the reach of stellar surface observation either by spectroscopic or photometric techniques. 


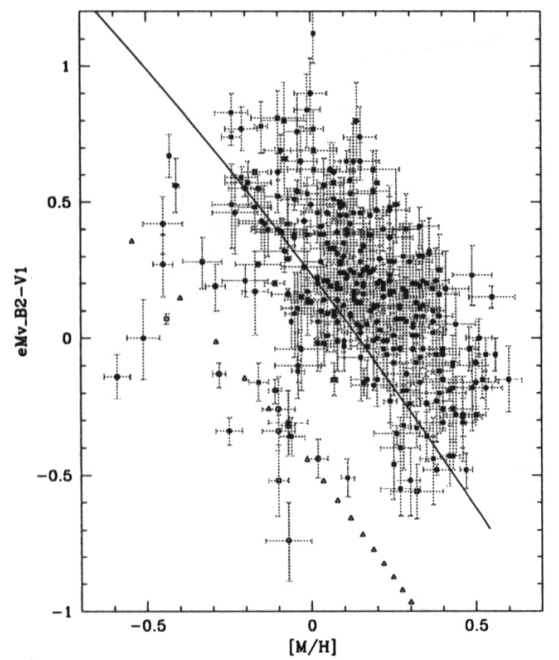

Figure 2. The departure from the Hyades main-sequence as a function of $\mathrm{Z}$, for $\mathrm{K}$ dwarfs in the $B 2-V 1$ colour interval $0.55-0.60$. The full line represents the mean relation for single field stars, the open triangles that for binaries with magnitude difference $=0.0$.

\section{The $T_{\text {eff }}$ scale problem}

The various $T_{\text {eff }}$ scales presently in use show systematic differences up to $300 \mathrm{~K}$ in the range 4000 to $7500 \mathrm{~K}$ and even more outside this range. Errors in $T_{\text {eff }}$ do not affect photometric $[\mathrm{M} / \mathrm{H}]$ but they do affect spectroscopic abundance determinations when photometric temperatures are used, e.g. $\Delta[\mathrm{Fe} / \mathrm{H}] \sim+0.10$ dex for $\Delta T_{\text {eff }}=+100 \mathrm{~K}$ for solar temperature stars. Errors in age determinations are more severe as $\Delta$ age $=1 \mathrm{Gyr}$ for $\Delta T_{\text {eff }}=40 \mathrm{~K}$ at the turn-off of 12 Gyr isochrones. Presently the best temperature determinations from the IRFM method show $\sigma T_{\text {eff }}=125 \mathrm{~K}$, with large systematic errors for late type stars with strong $\mathrm{TiO}$ bands. Intermediate band photometry is potentially able to provide $T_{\text {eff }}$ with errors as small as $20 \mathrm{~K}$ around $5000 \mathrm{~K}$. Until the temperature scale, the knowledge of stellar interiors and the model atmospheres are improved by half an order of magnitude, Hipparcos results will remain underexploited.

\section{References}

Girardi, L., Groenewegen, M.A.T., Weiss, A. \& Salaris, M. 1998, MNRAS, 301, 149

Gratton, R.G. 1998, MNRAS, 296, 739

van Leeuwen, F. \& Hansen Ruiz, C.S. 1997, ESA SP-402, 689

Mermilliod, J.-C. 2000, in "Very Low-Mass Stars and Brown Dwarfs", ed. R. Rebolo (Cambridge: Cambridge Univ. Press), in press

Twarog, B.A., Anthony-Twarog, B.J., \& Bricker, A.R. 1999, AJ, 117, 1816 